\title{
Ciclagem de nutrientes por plantas de cobertura na entressafra em um solo de cerrado
}

\author{
Carlo Adriano Boer(1), Renato Lara de Assis ${ }^{(2)}$, Gilson Pereira Silva(2), Antonio Joaquim Braga Pereira Braz ${ }^{(2)}$, \\ Alberto Leão de Lemos Barroso ${ }^{(2)}$, Alberto Cargnelutti Filho( ${ }^{(3)}$ e Fábio Ribeiro Pires ${ }^{(4)}$
}

\begin{abstract}
(1)Monsanto do Brasil, Dep. de Desenvolvimento Tecnológico, Av. Nações Unidas, no 1.2901, CEP 04578-910 São Paulo, SP. E-mail: carlo.a.boer@monsanto.com (2)Universidade de Rio Verde, Fac. de Agronomia, Caixa Postal 104, CEP $75901-970$ Rio Verde, GO. E-mail: assis@fesurv.br, gilson@fesurv.br, braga@fesurv.br, barroso@fesurv.br(3)Universidade Federal do Rio Grande do Sul, Dep. de Estatística, Av. Bento Gonçalves, no 9.500, CEP 91509-900 Porto Alegre, RS. E-mail: cargnelutti@ufrgs.br (4)Universidade Federal do Espírito Santo, Dep. de Ciências da Saúde, Biológicas e Agrárias, CEP 29933-415 São Mateus, ES. E-mail: fabiopires@ceunes.ufes.br
\end{abstract}

Resumo - O objetivo deste trabalho foi avaliar o acúmulo e a liberação de nutrientes (N, P, K, Ca, Mg e S) de resíduos culturais de plantas de cobertura na entressafra, em condições de Cerrado. $\mathrm{O}$ experimento foi conduzido em um Latossolo Vermelho distroférrico com textura argilosa. As plantas de cobertura avaliadas foram: amaranto (Amaranthus cruentus L.), milheto (Pennisetum glaucum L.) e capim-pé-de-galinha (Eleusine coracana (L.) Gaertn.). O delineamento experimental utilizado foi o de blocos ao acaso, no esquema de parcelas subdivididas, com quatro repetições. Na fase de florescimento das espécies, foi avaliada a produção de matéria seca e o acúmulo de nutrientes. A fim de avaliar a liberação de nutrientes dos resíduos culturais, o material vegetal de cada espécie foi acondicionado em sacolas de náilon, as quais foram dispostas sobre o solo e seu conteúdo analisado em intervalos de 30 dias, até 240 dias após sua instalação. As maiores quantidades de nutrientes acumulados na fitomassa das plantas de cobertura foram observadas no milheto e no capim-pé-de-galinha. O potássio foi o nutriente acumulado em maior quantidade, chegando a atingir 416,9 kg ha-1 no milheto. As maiores taxas de liberação de nutrientes foram observadas nos resíduos culturais do amaranto.

Termos para indexação: Amaranthus cruentus, Pennisetum glaucum, Eleusine coracana, dinâmica de decomposição, liberação de nutrientes, plantio direto.

\section{Nutrient cycling in off-season cover crops on a Brazilian savanna soil}

\begin{abstract}
The objective of this work was to evaluate the accumulation and the liberation of nutrients (N, P, K, $\mathrm{Ca}, \mathrm{Mg}$ and $\mathrm{S}$ ) of cultural residues by three species of cover crops, in off-season. Tested cover crops were amaranthus (Amaranthus cruentus L.), pearl millet (Pennisetum glaucum L.) and finger millet (Eleusine coracana (L.) Gaertn.). The experiment was carried out in a Typic Haplorthox clay texture soil. A randomized block desing in a split-plot array in time, with four replications, was used. At the flowering of the species, the production of dry matter and the accumulation of nutrients were evaluated. Proportional samples of dry matter of each cover crop species were placed in litter bags, which were distributed on the field plots surface, collected and weighed every 30 days, until 240 days after installation of the bags. The largest amounts of accumulated nutrients in the dry matter of the cover crops were observed with the pearl millet and the finger millet. The potassium was the nutrient accumulated in larger amount, reaching $416.9 \mathrm{~kg} \mathrm{ha}^{-1}$, in pearl millet. The largest rates of nutrient liberation were observed in the cultural residues of the amaranthus.
\end{abstract}

Index terms: Amaranthus cruentus, Pennisetum glaucum, Eleusine coracana, decomposition dynamics, nutrient release, no-till.

\section{Introdução}

A utilização e a ocupação agrícola da Região do Cerrado vêm ocorrendo com necessidade de adoção de novas tecnologias fundamentadas em bases conservacionistas. No sistema plantio direto, o uso de plantas de cobertura é uma alternativa para aumentar a sustentabilidade dos sistemas agrícolas, podendo restituir quantidades consideráveis de nutrientes aos cultivos, uma vez que essas plantas absorvem nutrientes das camadas subsuperficiais do solo e os liberam, posteriormente, na camada superficial pela decomposição dos seus resíduos (Duda et al., 2003). 
A utilização de culturas na entressafra com o objetivo de cobertura do solo e ciclagem de nutrientes, visando à diversificação da produção agrícola com sustentabilidade (Chaves \& Calegari, 2001), é uma estratégia para melhoria da qualidade ambiental, e diminui os efeitos nocivos do monocultivo.

Resíduos culturais na superfície do solo constituem importante reserva de nutrientes, cuja disponibilização pode ser rápida e intensa (Rosolem et al., 2003), ou lenta e gradual, conforme a interação entre os fatores climáticos, principalmente precipitação pluvial e temperatura, atividade macro e microbiológica do solo e qualidade e quantidade do resíduo vegetal (Alcântara et al., 2000; Oliveira et al., 2002). Diversas espécies de plantas de cobertura do solo podem ser utilizadas a fim de evitar sua exaustão. Porém, para que uma espécie seja eficaz na ciclagem de nutrientes, deve haver sincronia entre o nutriente liberado pelo resíduo da planta de cobertura e a demanda da cultura de interesse comercial, cultivada em sucessão (Braz et al., 2004).

Sob condições de Cerrado, as gramíneas têm desempenhado uma importante atuação como planta de cobertura, com destaque para o milheto. Sua utilização se deve à resistência ao déficit hídrico, elevada produção de biomassa e menor custo das sementes (Braz et al., 2004; Silva et al., 2006).

O não-revolvimento do solo e a manutenção da palhada em superfície resultam em menor velocidade de decomposição e menor liberação de nutrientes, quando comparados ao revolvimento do solo e à incorporação da palhada. A fim de descrever a liberação de nutrientes pela palhada, o modelo mais freqüentemente usado é o exponencial simples, descrito por Wieder \& Lang (1982) e utilizado por Moraes (2001) e Torres et al. (2005) que, por meio de uma constante, caracteriza a liberação de nutrientes e facilita as comparações de resultados.

A velocidade de decomposição bem como o acúmulo de nutrientes na biomassa e sua liberação variam entre as gramíneas e leguminosas. Moraes (2001) observou que a mineralização dos nutrientes no milheto e no sorgo, no inverno, ocorreu com mais intensidade nos primeiros 63 dias após a dessecação, ao passo que Torres et al. (2005), em estudo realizado na primavera, observaram maior acúmulo de $\mathrm{N}$ na crotalária e guandu, entretanto com maiores taxas de liberação de $\mathrm{N}$ até 43 dias após a dessecação. Na utilização da soja para fins de adubação verde em diferentes estádios de desenvolvimento, Padovani et al. (2006) observaram que a mineralização do $\mathrm{N}$ dos resíduos vegetais demorou de 20 a 34 dias para atingir a meia-vida. Trabalhos demonstram que as leguminosas apresentam maiores taxas iniciais de liberação de nutrientes, fato que pode ser explicado principalmente pela baixa relação $\mathrm{C} / \mathrm{N}$ do material.

Maior conhecimento da liberação de nutrientes da biomassa de espécies cultivadas que antecedem a cultura comercial é necessário. Há carência de informações sobre o processo de mineralização de nutrientes durante um período mais prolongado, no sistema de plantio direto com espécies alternativas, adaptadas às condições edafoclimáticas do Cerrado, como o amaranto e o capimpé-de-galinha.

O conhecimento da dinâmica de liberação dos nutrientes é fundamental para que se possa compatibilizar a máxima persistência dos resíduos culturais na superfície do solo, que contribui com a manutenção da umidade e com a proteção do solo contra efeitos erosivos.

O objetivo deste trabalho foi determinar o acúmulo e a liberação de nutrientes (N, P, K, Ca, Mg e S) na biomassa de amaranto, milheto e capim-pé-de-galinha, cultivados na entressafra, em solo do Cerrado.

\section{Material e Métodos}

$\mathrm{O}$ experimento foi instalado na área experimental da Universidade de Rio Verde, no Município de Rio Verde, GO (17047'30"S, 50 $57^{\circ} 44^{\prime \prime} \mathrm{W}$ e altitude de $770 \mathrm{~m}$ ), de abril de 2004 a março de 2005, em um Latossolo Vermelho distroférrico (Embrapa, 1999) de textura argilosa, com as seguintes características na camada arável $(0-20 \mathrm{~cm})$ por ocasião da instalação do experimento: $\mathrm{pH} \mathrm{em} \mathrm{CaCl}_{2}$ de 4,7; $1,24 \mathrm{mg} \mathrm{dm}^{-3} \mathrm{de}$ P; 0,68 $\mathrm{cmol}_{\mathrm{c}} \mathrm{dm}^{-3}$ de K; 3,02 $\mathrm{cmol}_{\mathrm{c}} \mathrm{dm}^{-3}$ de Ca; $1,22 \mathrm{cmol}_{\mathrm{c}} \mathrm{dm}^{-3}$ de $\mathrm{Mg} ; 4,90 \mathrm{cmol}_{\mathrm{c}} \mathrm{dm}^{-3}$ de $\mathrm{H}+\mathrm{Al}$; $21,8 \mathrm{~g} \mathrm{~kg}^{-1}$ de matéria orgânica e $50 \%$ de saturação por bases. A área experimental estava sob o sistema de plantio direto há três anos, com o plantio da soja no verão e milho na entressafra.

O delineamento utilizado foi o de blocos ao acaso, em esquema de parcelas subdivididas no tempo, com quatro repetições. As parcelas foram constituídas pelas espécies de plantas de cobertura milheto (Pennisetum glaucum var. ADR500), amaranto (Amaranthus cruentus, cultivar BRS Alegria) e capim-pé-de-galinha (Eleusine coracana). As subparcelas foram constituídas por nove épocas de avaliação: $0,30,60,90,120,150,180$, 210 e 240 dias após o manejo (dessecação). 
Antes da implantação das plantas de cobertura, realizou-se o controle das plantas daninhas presentes na área com herbicida. A abertura dos sulcos de plantio e adubação de semeadura foram realizadas mecanicamente, com semeadora-adubadora, aplicandose $250 \mathrm{~kg} \mathrm{ha}^{-1}$ da fórmula $8-16-16$. A semeadura foi realizada manualmente, em 17 de abril de 2004, utilizando-se $5 \mathrm{~kg} \mathrm{ha}^{-1}$ de sementes de amaranto, $10 \mathrm{~kg} \mathrm{ha}^{-1}$ de sementes de capim-pé-de-galinha e $12 \mathrm{~kg} \mathrm{ha}^{-1}$ de sementes de milheto. A área de cada parcela foi composta de quatro linhas com $4 \mathrm{~m}$ de comprimento, com espaçamento de 0,45 m entre linhas. As plantas de cobertura foram manejadas com o herbicida glyphosate na dosagem de $1.458 \mathrm{~g} \mathrm{ha}^{-1}$ de i.a., aos 59, 51 e 66 dias após o plantio do amaranto, milheto e capim-pé-de-galinha, respectivamente, quando cada espécie atingiu $50 \%$ de florescimento.

$\mathrm{Na}$ avaliação da produção de biomassa verde e seca, foi amostrada uma área de $0,25 \mathrm{~m}^{2}$ em três pontos ao acaso, em cada parcela, três dias após a dessecação, quando as plantas já apresentavam completa absorção do herbicida. O material amostrado foi secado em estufa com circulação e renovação forçada de ar, a $65^{\circ} \mathrm{C}$ por 72 horas.

A fim de avaliar a ciclagem de nutrientes, foram utilizadas sacolas de náilon ("litter bags") com malha de $2 \mathrm{~mm}$ de abertura, nas dimensões de $0,15 \times 0,20 \mathrm{~m}$, conforme proposto por Thomas \& Asakawa (1993). As sacolas foram instaladas na superfície do solo, na linha central da parcela, em número de oito por parcela. Em cada uma, foram colocadas diferentes quantidades de resíduos das plantas de cobertura, com 8,67, 32,4 e $26,26 \mathrm{~g}$, respectivamente, para amaranto, milheto e capim-pé-de-galinha, proporcionais à biomassa seca produzida por hectare, após o material vegetal ter sido picado em fragmentos de $5 \mathrm{~cm}$. As amostragens foram realizadas em intervalos regulares de 30 dias durante 240 dias. Os teores de N, P, K, Ca, Mg e S do tecido remanescente foram avaliados no florescimento e nos resíduos vegetais existentes nas sacolas de náilon aos $30,60,120,180$ e 240 dias, segundo método proposto por Malavolta et al. (1997). O carbono (C) foi determinado na época do florescimento, pela queima em mufla à temperatura de $550^{\circ} \mathrm{C}$ (Embrapa, 1997). Foram calculadas as quantidades remanescentes dos nutrientes existentes nas sacolas de decomposição em cada época. Os resultados foram submetidos à análise de variância. As médias foram comparadas pelo teste de Tukey, a $5 \%$ de probabilidade.
A fim de descrever a liberação de nutrientes, os dados foram ajustados a um modelo matemático exponencial, descrito por Wieder \& Lang (1982): Q = $\mathrm{Q}_{0} \exp (-\mathrm{kt})$; em que $\mathrm{Q}$ é a quantidade de nutrientes existente no tempo t $\left(\mathrm{kg} \mathrm{ha}^{-1}\right) ; \mathrm{Q}_{\mathrm{o}}$ é a fração de nutrientes potencialmente liberados $\left(\mathrm{kg} \mathrm{ha}^{-1}\right)$; e k é a taxa de liberação dos nutrientes $\left(\mathrm{g} \mathrm{g}^{-1}\right)$. Com o valor de $\mathrm{k}$, foi calculado o tempo de meia-vida ( $\mathrm{T}_{1 / 2}$ vida) dos nutrientes nos resíduos culturais remanescentes, ou seja, o tempo necessário para que $50 \%$ dos nutrientes sejam liberados. Nesse cálculo, utilizou-se a fórmula $T_{1 / 2}=0,693 / \mathrm{k}$ proposta por Paul \& Clark (1989).

As análises de regressão relativas à liberação de nutrientes foram realizadas com uso do aplicativo Sigma Plot, versão 7.0, da Jandel Scientific, e, nas comparações das equações de regressão após linearização, foi utilizado o procedimento descrito em Snedecor \& Cochran (1989).

\section{Resultados e Discussão}

Na Figura 1, encontram-se os dados referentes à temperatura média mensal e precipitação total mensal durante o período de condução do experimento.

A produção de biomassa seca foi de 2.891, $10.801 \mathrm{e}$ $8.753 \mathrm{~kg} \mathrm{ha}^{-1}$, e uma relação $\mathrm{C} / \mathrm{N}$ de 21,34 e 29 , respectivamente, para amaranto, milheto e capim-péde-galinha. O milheto e o capim-pé-de-galinha se destacaram na produção de biomassa. Os valores alcançados no milheto foram próximos aos encontrados por Torres et al. (2005), ao passo que no capim-pé-de-

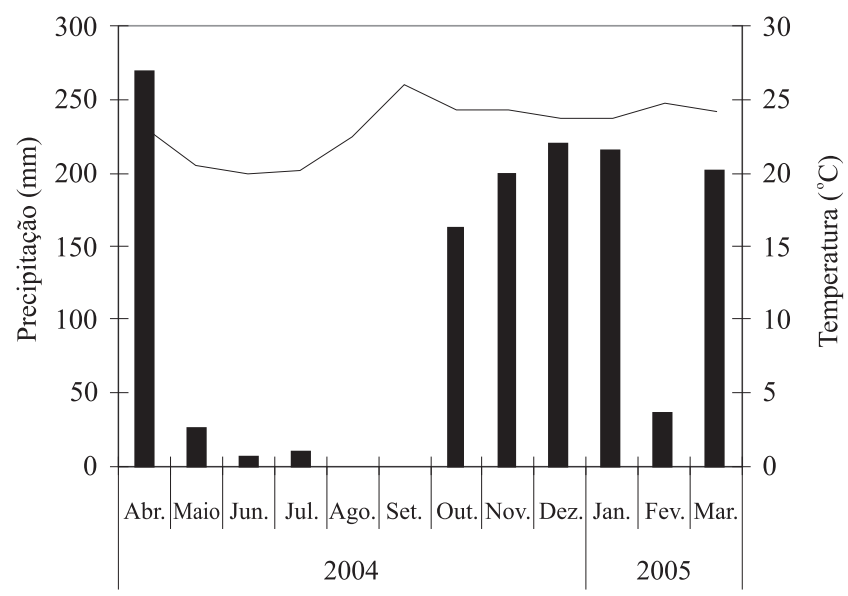

Figura 1. Precipitação total mensal (-) e temperatura média mensal $(-)$ no período de condução do experimento. 
galinha, os resultados foram superiores aos de Francisco (2002). Essa diferença de produtividade está relacionada com as condições climáticas, de solo e com as cultivares utilizadas.

O maior acúmulo de $\mathrm{Ne} \mathrm{Mg}$ foi verificado na palhada do capim-pé-de-galinha, e o de K, na palhada do milheto. Milheto e capim-pé-de-galinha acumularam quantidades maiores de $\mathrm{P}, \mathrm{Ca}$ e $\mathrm{S}$ que o amaranto, o que demonstra o grande potencial de acúmulo dessas espécies (Tabela 1). A quantidade de nutrientes acumulada depende da espécie utilizada, do estádio fenológico, da produção de matéria seca e do período de plantio.

Braz et al. (2004) observaram grande acúmulo de $\mathrm{N}$ (348 $\left.\mathrm{kg} \mathrm{ha}^{-1}\right)$ e K (314 $\left.\mathrm{kg} \mathrm{ha}^{-1}\right)$ no milheto BN2, quando o plantio foi realizado no verão, em Região do Cerrado, com elevado índice pluvial e elevadas temperaturas. Neste trabalho, as elevadas precipitações verificadas em abril e início de maio, associadas à elevada temperatura de abril (Figura 1), favoreceram maior acúmulo de biomassa e nutrientes, principalmente pelo milheto e pelo capim-pé-de-galinha.

Tabela 1. Nutrientes remanescentes na biomassa das palhadas de amaranto (AM), milheto (MI) e capim-pé-de-galinha (CPG), até 240 dias após o manejo ${ }^{(1)}$

\begin{tabular}{|c|c|c|c|c|c|c|}
\hline \multirow[t]{2}{*}{ Espécie } & \multicolumn{6}{|c|}{ Dias após o manejo } \\
\hline & 0 & 30 & 60 & 120 & 180 & 240 \\
\hline & \multicolumn{6}{|c|}{ Nitrogênio $\left(\mathrm{kg} \mathrm{ha}^{-1}\right)$} \\
\hline $\mathrm{AM}$ & $49,36 \mathrm{c}$ & $20,17 b$ & $12,03 b$ & $4,78 \mathrm{c}$ & $3,69 \mathrm{c}$ & $2,96 b$ \\
\hline MI & $121,75 b$ & $112,65 \mathrm{a}$ & $98,98 \mathrm{a}$ & $77,26 \mathrm{a}$ & $32,86 a$ & $14,67 \mathrm{a}$ \\
\hline \multirow[t]{2}{*}{ CPG } & $133,24 a$ & $102,35 \mathrm{a}$ & $92,66 a$ & $51,33 \mathrm{~b}$ & $16,56 \mathrm{~b}$ & $11,47 \mathrm{ab}$ \\
\hline & \multicolumn{6}{|c|}{ Fósforo $\left(\mathrm{kg} \mathrm{ha}^{-1}\right)$} \\
\hline $\mathrm{AM}$ & $7,61 b$ & $2,57 b$ & $1,40 b$ & $0,50 \mathrm{c}$ & $0,44 b$ & $0,38 \mathrm{a}$ \\
\hline MI & $17,26 \mathrm{a}$ & $11,59 \mathrm{a}$ & $9,82 \mathrm{a}$ & $9,22 \mathrm{a}$ & $3,84 \mathrm{a}$ & $1,66 \mathrm{a}$ \\
\hline \multirow[t]{2}{*}{$\mathrm{CPG}$} & $18,26 \mathrm{a}$ & $11,45 \mathrm{a}$ & $9,51 \mathrm{a}$ & $6,54 b$ & $1,86 \mathrm{ab}$ & $1,62 \mathrm{a}$ \\
\hline & \multicolumn{6}{|c|}{ Potássio $\left(\mathrm{kg} \mathrm{ha}^{-1}\right)$} \\
\hline $\mathrm{AM}$ & $139,78 \mathrm{c}$ & $15,97 b$ & $2,16 b$ & $0,28 \mathrm{a}$ & $0,25 \mathrm{a}$ & $0,19 \mathrm{a}$ \\
\hline MI & $416,94 a$ & $102,06 a$ & $51,90 \mathrm{a}$ & $22,33 a$ & $2,58 \mathrm{a}$ & $0,73 a$ \\
\hline \multirow[t]{2}{*}{ CPG } & $174,59 \mathrm{~b}$ & $77,77 \mathrm{a}$ & $41,53 \mathrm{a}$ & $6,90 \mathrm{a}$ & $2,22 \mathrm{a}$ & $0,72 \mathrm{a}$ \\
\hline & \multicolumn{6}{|c|}{ Cálcio (kg ha-1) } \\
\hline $\mathrm{AM}$ & $57,88 \mathrm{~b}$ & $42,42 b$ & $37,54 b$ & $27,98 \mathrm{a}$ & $11,02 \mathrm{a}$ & $5,01 \mathrm{a}$ \\
\hline MI & $76,31 \mathrm{a}$ & $60,13 \mathrm{a}$ & $49,05 \mathrm{a}$ & $28,10 \mathrm{a}$ & $8,81 \mathrm{a}$ & $4,94 a$ \\
\hline \multirow[t]{2}{*}{$\mathrm{CPG}$} & $78,32 \mathrm{a}$ & $33,22 \mathrm{c}$ & $26,07 \mathrm{c}$ & $9,54 b$ & $6,78 \mathrm{a}$ & $4,06 \mathrm{a}$ \\
\hline & \multicolumn{6}{|c|}{ Magnésio $\left(\mathrm{kg} \mathrm{ha}^{-1}\right)$} \\
\hline $\mathrm{AM}$ & $32,81 b$ & $16,53 b$ & $8,45 b$ & $2,01 \mathrm{c}$ & $1,82 \mathrm{a}$ & $0,99 a$ \\
\hline MI & $39,76 b$ & $34,07 \mathrm{a}$ & $29,14 \mathrm{a}$ & $25,47 \mathrm{a}$ & $9,91 \mathrm{a}$ & $4,30 \mathrm{a}$ \\
\hline \multirow[t]{2}{*}{ CPG } & $50,94 \mathrm{a}$ & $33,51 \mathrm{a}$ & $27,09 \mathrm{a}$ & $14,04 \mathrm{~b}$ & $4,47 \mathrm{a}$ & $3,13 \mathrm{a}$ \\
\hline & \multicolumn{6}{|c|}{ Enxofre $\left(\mathrm{kg} \mathrm{ha}^{-1}\right)$} \\
\hline $\mathrm{AM}$ & $6,68 b$ & $2,75 b$ & $1,25 b$ & $0,63 b$ & $0,57 \mathrm{a}$ & $0,48 \mathrm{a}$ \\
\hline MI & $17,90 \mathrm{a}$ & $14,06 \mathrm{a}$ & $9,81 \mathrm{a}$ & $8,76 a$ & $3,78 \mathrm{a}$ & $1,72 \mathrm{a}$ \\
\hline $\mathrm{CPG}$ & $18,36 \mathrm{a}$ & $13,06 \mathrm{a}$ & $10,13 \mathrm{a}$ & $6,75 a$ & $2,53 \mathrm{a}$ & $2,22 \mathrm{a}$ \\
\hline
\end{tabular}

${ }^{(1)}$ Médias seguidas pela mesma letra na coluna, para cada nutriente, não diferem entre si pelo teste de Tukey, a $5 \%$ de probabilidade.
As quantidades de $\mathrm{N}, \mathrm{P}, \mathrm{Ca}$ e $\mathrm{S}$ observadas na biomassa seca de milheto, por ocasião do florescimento (Tabela 1), foram inferiores às encontradas por Oliveira et al. (2002), 100 dias após o plantio, e Moraes (2001), no florescimento, que registraram, respectivamente, 162,7 e $126,7 \mathrm{~kg} \mathrm{ha}^{-1}$ de N; 24,8 e $23,1 \mathrm{~kg} \mathrm{ha}^{-1}$ de P; 93,3 e 105,6 kg ha-1 de Ca e 25,6 e 22,9 $\mathrm{kg} \mathrm{ha}^{-1}$ de S, em 14.180 e $9.630 \mathrm{~kg} \mathrm{ha}^{-1}$ de biomassa seca de milheto comum.

O acúmulo de $\mathrm{N}$ no milheto neste trabalho foi superior ao encontrado por Torres et al. (2005), que obtiveram, com $3.600 \mathrm{~kg} \mathrm{ha}^{-1}$ de biomassa seca, 55,8 $\mathrm{kg} \mathrm{ha}^{-1} \mathrm{de} \mathrm{N}$. Quanto ao Mg, o acúmulo desse nutriente no milheto foi superior aos obtidos por Teixeira et al. (2005) e Moraes (2001), porém inferior ao obtido por Oliveira et al. (2002). Quanto ao K, os 416,9 $\mathrm{kg} \mathrm{ha}^{-1}$ acumulados pelo milheto ADR500 (Tabela 1) foram superiores aos encontrados por Teixeira et al. (2005), Oliveira et al. (2002) e Moraes (2001), em milheto comum, os quais obtiveram, respectivamente, $50,9,20,8$ e $166,0 \mathrm{~kg} \mathrm{ha}^{-1}$ de $\mathrm{K}$, com produção de 2.907, 14.180 e $9.630 \mathrm{~kg} \mathrm{ha}^{-1}$ de biomassa seca. $\mathrm{O}$ acúmulo de $\mathrm{N}$ e, principalmente, do potássio, no milheto ADR500, se deve ao fato de a cultivar utilizada ser resultante de um programa de melhoramento genético e possuir grande capacidade de produção de biomassa, ciclagem e acúmulo de nutrientes. Quanto aos demais autores, realizaram estudos com o milheto comum, com menor potencial genético.

Os teores de $\mathrm{N}, \mathrm{P}, \mathrm{K}, \mathrm{Ca}, \mathrm{Mg}$ e $\mathrm{S}$ encontrados no florescimento no capim-pé-de-galinha foram superiores aos encontrados por Francisco (2002), que obteve, em $4.364 \mathrm{~kg} \mathrm{ha}^{-1}$ de biomassa seca, $85,3 \mathrm{~kg} \mathrm{ha}^{-1}$ de $\mathrm{N}$, 6,5 $\mathrm{kg} \mathrm{ha}^{-1}$ de P, 101,5 kg ha-1 de K, 30,5 kg ha-1 de Ca, 14,6 $\mathrm{kg} \mathrm{ha}^{-1}$ de $\mathrm{Mg}$ e 10,6 $\mathrm{kg} \mathrm{ha}^{-1}$ de $\mathrm{S}$, aos 81 dias após o plantio. Ainda no capim-pé-de-galinha, e também no amaranto, o N, o P e o Ca acumulados na biomassa, neste trabalho, foram superiores aos encontrados por Azevedo \& Nascimento (2002), que obtiveram, 32,7 e $14,10 \mathrm{~kg} \mathrm{ha}^{-1}$ de $\mathrm{N}, 4,05$ e $2,56 \mathrm{~kg} \mathrm{ha}^{-1}$ de $\mathrm{P}$ e $7,51 \mathrm{e}$ $3,29 \mathrm{~kg} \mathrm{ha}^{-1} \mathrm{de} \mathrm{Ca}$, respectivamente, em $2.890 \mathrm{e}$ $1.830 \mathrm{~kg} \mathrm{ha}^{-1}$ de biomassa seca remanescente após a colheita dos grãos.

Em estudos sobre plantas de cobertura, observa-se que a quantidade de nutrientes acumulados depende da espécie, da fertilidade do solo, do estádio fenológico na dessecação, da relação $\mathrm{C} / \mathrm{N}$, da época de plantio além das condições climáticas de cada estudo (Primavesi et al., 2002). Ao se comparar os dados deste trabalho 
com os da literatura, observa-se que o milheto se destaca como cultura recicladora, com elevado acúmulo de nutrientes, principalmente de $\mathrm{N}$ e $\mathrm{K}$.

$\mathrm{Na}$ análise da liberação de nutrientes da biomassa das plantas de cobertura, observa-se que a maior velocidade de liberação de $\mathrm{P}$ e $\mathrm{K}$ ocorreu até os 30 dias após a dessecação (Tabela 2). Observa-se que esses nutrientes apresentaram as maiores taxas de liberação aos 30 dias, com valores de 66,2, 32,9 e 37,3\% para o $P$ e $88,6,75,5$ e $55,5 \%$ para o $\mathrm{K}$, respectivamente, relativos ao amaranto, milheto e capim-pé-de-galinha. No caso do $\mathrm{P}$, que se encontra associado a compostos orgânicos no tecido vegetal (Marschner, 1995), sua mineralização está ligada ao processo de decomposição pelos microrganismos do solo. A mineralização mais rápida no amaranto se deve à sua menor relação $\mathrm{C} / \mathrm{N}$.

No milheto, a liberação de N, P, Ca, Mg e S, aos 180 dias após o manejo, foi inferior à encontrada por Moraes (2001) que, com milheto comum, obteve, para esses nutrientes, aos 168 dias após o manejo, liberação de 96, 93,2, 97,6, 87,6 e 81,6\%, respectivamente. Esta mineralização mais lenta também pode ser atestada pela

Tabela 2. Porcentagem de nutrientes liberados da biomassa nas palhadas de amaranto (AM), milheto (MI) e capim-pé-de-galinha (CPG), até 240 dias após o manejo.

\begin{tabular}{|c|c|c|c|c|c|}
\hline \multirow[t]{2}{*}{ Espécie } & \multicolumn{5}{|c|}{ Dias após o manejo } \\
\hline & 30 & 60 & 120 & 180 & 240 \\
\hline & \multicolumn{5}{|c|}{ Nitrogênio } \\
\hline AM & 59,1 & 75,6 & 90,3 & 92,5 & 94,0 \\
\hline MI & 7,5 & 18,7 & 36,5 & 73,0 & 88,0 \\
\hline \multirow[t]{2}{*}{$\mathrm{CPG}$} & 23,2 & 30,5 & 61,5 & 87,6 & 91,4 \\
\hline & \multicolumn{5}{|c|}{ Fósforo } \\
\hline $\mathrm{AM}$ & 66,2 & 81,6 & 93,4 & 94,2 & 95,0 \\
\hline MI & 32,9 & 43,1 & 46,6 & 77,8 & 90,4 \\
\hline \multirow[t]{2}{*}{$\mathrm{CPG}$} & 37,3 & 47,9 & 64,2 & 89,8 & 91,1 \\
\hline & \multicolumn{5}{|c|}{ Potássio } \\
\hline $\mathrm{AM}$ & 88,6 & 98,5 & 99,8 & 99,8 & 99,9 \\
\hline MI & 75,5 & 87,6 & 94,6 & 99,4 & 99,8 \\
\hline \multirow[t]{2}{*}{$\mathrm{CPG}$} & 55,5 & 76,2 & 96,0 & 98,7 & 99,6 \\
\hline & \multicolumn{5}{|c|}{ Cálcio } \\
\hline AM & 26,7 & 35,1 & 51,7 & 81,0 & 91,3 \\
\hline MI & 21,2 & 35,7 & 63,2 & 88,5 & 93,5 \\
\hline \multirow[t]{2}{*}{$\mathrm{CPG}$} & 57,6 & 66,7 & 87,8 & 91,3 & 94,8 \\
\hline & \multicolumn{5}{|c|}{ Magnésio } \\
\hline $\mathrm{AM}$ & 49,6 & 74,2 & 93,9 & 94,5 & 97,0 \\
\hline MI & 14,3 & 26,7 & 35,9 & 75,1 & 89,2 \\
\hline \multirow[t]{2}{*}{$\mathrm{CPG}$} & 34,2 & 46,8 & 72,4 & 91,2 & 93,9 \\
\hline & \multicolumn{5}{|c|}{ Enxofre } \\
\hline $\mathrm{AM}$ & 58,8 & 81,3 & 90,6 & 91,5 & 92,8 \\
\hline MI & 21,5 & 45,2 & 51,1 & 78,9 & 90,4 \\
\hline $\mathrm{CPG}$ & 28,9 & 44,8 & 63,2 & 86,2 & 87,9 \\
\hline
\end{tabular}

análise da meia-vida desses nutrientes (Tabela 3). Com os dados obtidos para os nutrientes remanescentes na palhada de milheto no trabalho de Moraes (2001), observou-se meia-vida de 89 dias para N, 43 dias para P, 27 dias para $\mathrm{Ca}, 56$ dias para $\mathrm{Mg}$ e 69 dias para $\mathrm{S}$. Neste trabalho, a maior meia-vida desses nutrientes pode ser justificada pela menor pluviosidade e temperaturas mais baixas nos primeiros meses após dessecação (Figura 1), que propiciaram menor decomposição da biomassa seca remanescente e conseqüentemente menor liberação de nutrientes.

Nas três espécies, o K foi o nutriente mais rapidamente mineralizado (Tabelas 1 e 2). A maior velocidade de liberação desse nutriente também pode ser atestada pela análise da meia-vida, de 10, 16 e 27 dias para amaranto, milheto e capim-pé-de-galinha (Tabela 3), respectivamente, e pode ser atribuída ao fato de o $\mathrm{K}$ ser um elemento que não está associado a nenhum componente estrutural do tecido vegetal e encontrar-se na forma iônica (Marschner,

Tabela 3. Coeficientes da equação de regressão $\mathrm{Q}=\mathrm{Q}_{0} \cdot \exp (-\mathrm{kt})$, e meia-vida, para $\mathrm{N}, \mathrm{P}, \mathrm{K}, \mathrm{Ca}, \mathrm{Mg}$ e $\mathrm{S}$ remanescentes até 240 dias após a dessecação.

\begin{tabular}{|c|c|c|c|}
\hline Parâmetro & Amaranto & Milheto & Capim-pé-de-galinha \\
\hline & \multicolumn{3}{|c|}{ Nitrogênio } \\
\hline $\mathrm{Q}_{\mathrm{o}}\left(\mathrm{kg} \mathrm{ha}^{-1}\right)$ & 48,51 & 131,70 & 137,02 \\
\hline $\mathrm{k}\left(\mathrm{g} \mathrm{g}^{-1}\right)$ & 0,0250 & 0,0063 & 0,0089 \\
\hline $\mathrm{R}^{2}$ & 0,98 & 0,92 & 0,97 \\
\hline \multirow[t]{2}{*}{ Meia-vida (dias) } & 28 & 110 & 78 \\
\hline & \multicolumn{3}{|c|}{ Fósforo } \\
\hline $\mathrm{Q}_{\mathrm{o}}\left(\mathrm{kg} \mathrm{ha}^{-1}\right)$ & 7,54 & 16,38 & 17,55 \\
\hline $\mathrm{k}\left(\mathrm{g} \mathrm{g}^{-1}\right)$ & 0,0319 & 0,0075 & 0,0103 \\
\hline $\mathrm{R}^{2}$ & 0,98 & 0,93 & 0,97 \\
\hline \multirow[t]{2}{*}{ Meia-vida (dias) } & 22 & 92 & 67 \\
\hline & \multicolumn{3}{|c|}{ Potássio } \\
\hline $\mathrm{Q}_{\mathrm{o}}\left(\mathrm{kg} \mathrm{ha}^{-1}\right)$ & 139,78 & 415,04 & 173,90 \\
\hline $\mathrm{k}\left(\mathrm{g} \mathrm{g}^{-1}\right)$ & 0,0722 & 0,0428 & 0,0255 \\
\hline $\mathrm{R}^{2}$ & 1,00 & 0,99 & 0,99 \\
\hline \multirow[t]{2}{*}{ Meia-vida (dias) } & 10 & 16 & 27 \\
\hline & \multicolumn{3}{|c|}{ Cálcio } \\
\hline $\mathrm{Q}_{\mathrm{o}}\left(\mathrm{kg} \mathrm{ha}^{-1}\right)$ & 57,54 & 78,80 & 75,62 \\
\hline $\mathrm{k}\left(\mathrm{g} \mathrm{g}^{-1}\right)$ & 0,0079 & 0,0094 & 0,0204 \\
\hline $\mathrm{R}^{2}$ & 0,97 & 0,98 & 0,97 \\
\hline \multirow[t]{2}{*}{ Meia-vida (dias) } & 88 & 74 & 34 \\
\hline & \multicolumn{3}{|c|}{ Magnésio } \\
\hline $\mathrm{Q}_{\mathrm{o}}\left(\mathrm{kg} \mathrm{ha}^{-1}\right)$ & 32,73 & 41,47 & 50,34 \\
\hline $\mathrm{k}\left(\mathrm{g} \mathrm{g}^{-1}\right)$ & 0,0225 & 0,0065 & 0,0115 \\
\hline $\mathrm{R}^{2}$ & 0,99 & 0,92 & 0,99 \\
\hline \multirow[t]{2}{*}{ Meia-vida (dias) } & 31 & 107 & 60 \\
\hline & \multicolumn{3}{|c|}{ Enxofre } \\
\hline $\mathrm{Q}_{\mathrm{o}}\left(\mathrm{kg} \mathrm{ha}^{-1}\right)$ & 6,62 & 17,77 & 18,05 \\
\hline $\mathrm{k}\left(\mathrm{g} \mathrm{g}^{-1}\right)$ & 0,0273 & 0,0080 & 0,0094 \\
\hline $\mathrm{R}^{2}$ & 0,98 & 0,99 & 0,97 \\
\hline Meia-vida (dias) & 25 & 87 & 74 \\
\hline
\end{tabular}


1995; Bartz, 1998). Por esta razão, esse elemento pode ser facilmente extraído dos tecidos das plantas, tanto pela água da chuva quanto pela própria umidade do solo, pois no período de maior liberação do potássio praticamente não ocorreram precipitações (Figura 1). Dessa forma o K representa o nutriente em que o prazo de implantação de culturas em sucessão deve ser minimizado, com vistas à diminuição de perdas, devido a sua rápida liberação.

Os coeficientes das equações ajustadas para a descrição da liberação de nutrientes nas palhadas remanescentes estão apresentados na Tabela 3. Observou-se que foram semelhantes para milheto e capim-pé-de-galinha (Tabela 4), na liberação de N, P, $\mathrm{Mg}$ e S, podendo-se concluir que houve comportamento semelhante de liberação destes nutrientes nas duas espécies. A explicação está no fato de serem gramíneas e apresentarem relação $\mathrm{C} / \mathrm{N}$ próximas. Esse resultado demonstra o potencial de utilização dessas espécies como plantas de cobertura para a Região do Cerrado, principalmente na entressafra, já que além de acumularem biomassa, promovem a reciclagem de nutrientes e sua liberação gradativa.

Quanto aos valores de liberação de N, P, Mg e S de acordo com as datas de avaliação, como não haviam diferido no milheto e no capim-pé-de-galinha, foram ajustadas novas equações para milheto + capim-pé-degalinha (Tabela 5 e Figura 2). Nova equação foi ajustada também para amaranto e milheto na liberação de $\mathrm{Ca}$, o que demonstra, novamente, o comportamento semelhante de liberação desse nutriente nessas duas espécies (Tabela 5 e Figura 2). Quanto à liberação de $\mathrm{K}$, as três espécies apresentaram comportamento

Tabela 4. Comparação das equações de regressão (teste F), para N, P, K, Ca, Mg e S remanescentes até 240 dias após o manejo, para amaranto, milheto e capim-pé-de-galinha.

\begin{tabular}{lcccccc}
\hline Plantas de cobertura & $\mathrm{N}$ & $\mathrm{P}$ & $\mathrm{K}$ & $\mathrm{Ca}$ & $\mathrm{Mg}$ & $\mathrm{S}$ \\
\hline $\begin{array}{l}\text { Amaranto vs. milheto } \\
\text { Amaranto vs. }\end{array}$ & $* *$ & $* *$ & $* *$ & $\mathrm{~ns}$ & $* *$ & $* *$ \\
$\begin{array}{l}\text { capim-pé-de-galinha } \\
\text { Milheto vs. }\end{array}$ & $* *$ & $* *$ & $* *$ & $*$ & $* *$ & $* *$ \\
capim-pé-de-galinha & $\mathrm{ns}$ & $\mathrm{ns}$ & $*$ & $* *$ & $\mathrm{~ns}$ & $\mathrm{~ns}$ \\
$\begin{array}{l}\text { Recalculando o F para as equações que não diferiram estatisticamente } \\
\begin{array}{l}\text { Milheto + capim-pé-de- } \\
\text { galinha vs. amaranto }\end{array}\end{array}$ & $* *$ & $* *$ & & & $* *$ & $* *$ \\
$\begin{array}{l}\text { Amaranto + milheto vs. } \\
\text { capim-pé-de-galinha }\end{array}$ & & & & & & \\
\hline
\end{tabular}

nsNão-significativo. * e **Significativo a 5 e $1 \%$ de probabilidade, respectivamente. diferenciado (Tabela 4 e Figura 2). Na análise dos modelos ajustados aos dados obtidos em todas as épocas de amostragem, observa-se que o padrão de liberação de nutrientes apresenta duas fases distintas: a primeira ocorre de forma mais rápida (entre 0 e 120 dias) e é relativa à liberação dos nutrientes das folhas e dos materiais menos lignificados; a segunda é mais lenta (entre 120 e 240 dias) e é relativa à decomposição de compostos mais resistentes (Figura 2). Aita \& Giacomini (2003), ao avaliar a decomposição e liberação de $\mathrm{N}$ de resíduos culturais de plantas de cobertura, observaram comportamentos semelhantes para as gramíneas.

O comportamento semelhante na dinâmica de liberação de N, P, Mg e S entre o milheto e o capim-péde-galinha, e nos maiores valores obtidos em relação ao amaranto, até 240 dias após o manejo, demonstra que essas espécies são opções eficientes na ciclagem dos nutrientes no sistema plantio direto no Cerrado (Figura 2), em razão do maior acúmulo de biomassa e acúmulo de grande quantidade de nutrientes.

Os nutrientes presentes nos resíduos culturais do amaranto apresentam elevada taxa de liberação, ao contrário do observado para o milheto e para o capim-pé-de-galinha

Tabela 5. Coeficientes das novas equações de regressão em milheto + capim-pé-de-galinha para N, P, Mg e S e amaranto + milheto para $\mathrm{Ca}$, remanescentes até 240 dias após o manejo.

\begin{tabular}{|c|c|c|}
\hline Parâmetro & $\begin{array}{l}\text { Milheto + capim- } \\
\text { pé-de-galinha }\end{array}$ & $\begin{array}{l}\text { Amaranto + } \\
\text { milheto }\end{array}$ \\
\hline & \multicolumn{2}{|c|}{ Nitrogênio } \\
\hline $\mathrm{Q}_{\mathrm{o}}\left(\mathrm{kg} \mathrm{ha}^{-1}\right)$ & 133,9 & - \\
\hline $\mathrm{k}\left(\mathrm{g} \mathrm{g}^{-1}\right)$ & 0,0074 & - \\
\hline \multirow[t]{2}{*}{$\mathrm{R}^{2}$} & 0,93 & - \\
\hline & \multicolumn{2}{|c|}{ Fósforo } \\
\hline $\mathrm{Q}_{\mathrm{o}}\left(\mathrm{kg} \mathrm{ha}^{-1}\right)$ & 16,92 & - \\
\hline $\mathrm{k}\left(\mathrm{g} \mathrm{g}^{-1}\right)$ & 0,0088 & - \\
\hline \multirow[t]{2}{*}{$\mathrm{R}^{2}$} & 0,94 & - \\
\hline & \multicolumn{2}{|c|}{ Magnésio } \\
\hline $\mathrm{Q}_{\mathrm{o}}\left(\mathrm{kg} \mathrm{ha}^{-1}\right)$ & 45,42 & - \\
\hline $\mathrm{k}\left(\mathrm{g} \mathrm{g}^{-1}\right)$ & 0,0086 & - \\
\hline \multirow[t]{2}{*}{$\mathrm{R}^{2}$} & 0,93 & - \\
\hline & \multicolumn{2}{|c|}{ Enxofre } \\
\hline $\mathrm{Q}_{\mathrm{o}}\left(\mathrm{kg} \mathrm{ha}^{-1}\right)$ & 17,89 & - \\
\hline $\mathrm{k}\left(\mathrm{g} \mathrm{g}^{-1}\right)$ & 0,0087 & - \\
\hline \multirow[t]{2}{*}{$\mathrm{R}^{2}$} & 0,97 & - \\
\hline & \multicolumn{2}{|c|}{ Cálcio } \\
\hline $\mathrm{Q}_{\mathrm{o}}\left(\mathrm{kg} \mathrm{ha}^{-1}\right)$ & - & 68,16 \\
\hline $\mathrm{k}\left(\mathrm{g} \mathrm{g}^{-1}\right)$ & - & 0,0088 \\
\hline $\mathrm{R}^{2}$ & - & 0,92 \\
\hline
\end{tabular}



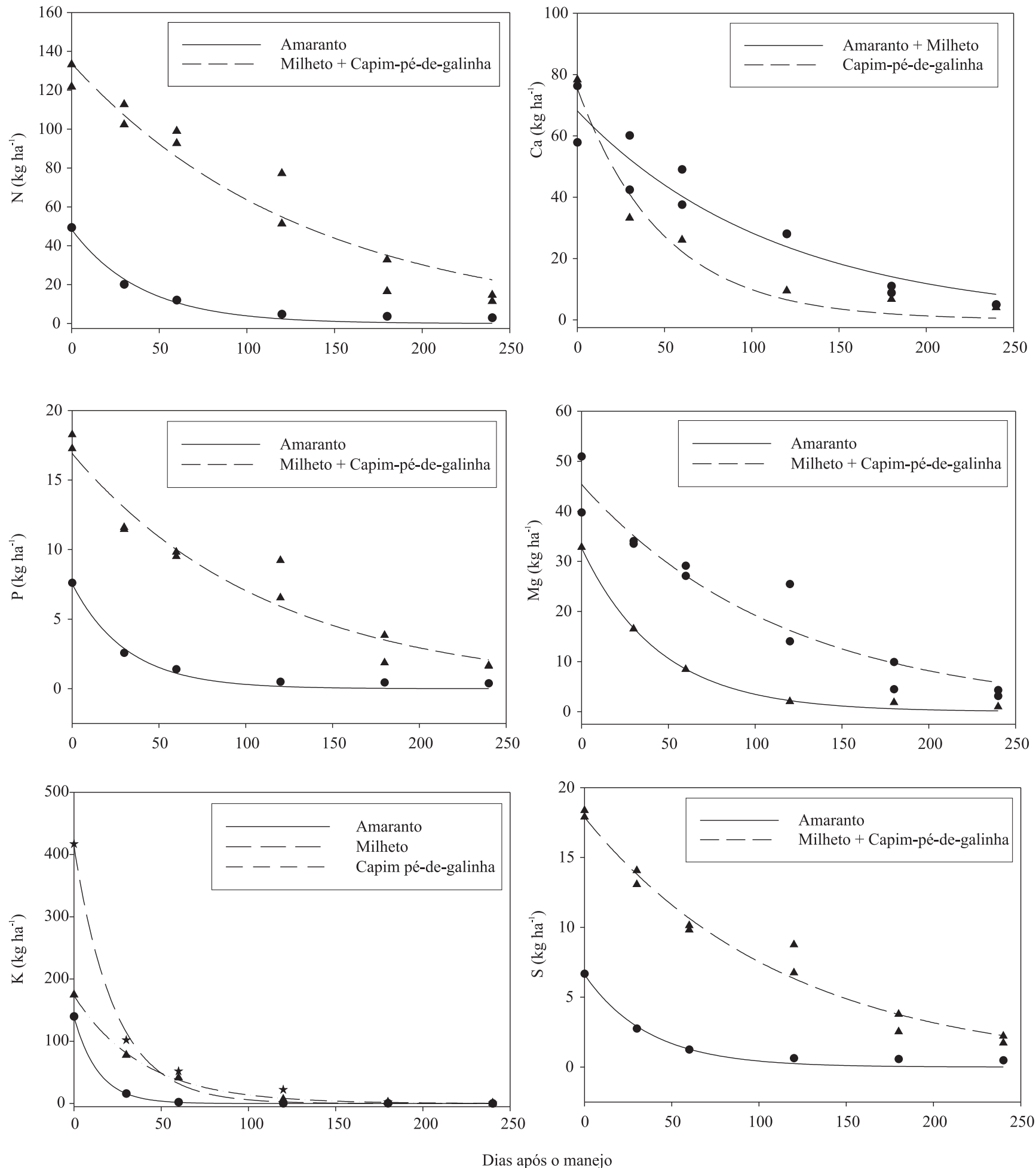

Figura 2. Nutrientes remanescentes nas biomassas das palhadas de amaranto, milheto e capim-pé-de-galinha até 240 dias após o manejo. 
(Tabela 2). A rápida liberação dos nutrientes no amaranto indica que os resíduos dessa espécie são rapidamente decompostos, em razão da baixa relação $\mathrm{C} / \mathrm{N}$ (21), o que diminui sua eficiência como planta de cobertura.

\section{Conclusões}

1. O milheto e o capim-pé-de-galinha acumulam as maiores quantidades de nutrientes na biomassa.

2. O potássio é o nutriente acumulado em maior quantidade.

3. A liberação de potássio é a mais rápida e apresenta comportamento diferenciado entre as espécies.

4. As maiores taxas de liberação de nutrientes são observadas nos resíduos culturais do amaranto.

\section{Referências}

AITA, C.; GIACOMINI, S.J. Decomposição e liberação de nitrogênio de resíduos culturais de plantas de cobertura de solo solteiras e consorciadas. Revista Brasileira de Ciência do Solo, v.27, p.601-612, 2003.

ALCÂNTARA, F.A.; FURTINI NETO, A.E.; PAULA, M.B. de; MESQUITA, H.A. de; MUNIZ, J.A. Adubação verde na recuperação da fertilidade de um Latossolo Vermelho-Escuro degradado. Pesquisa Agropecuária Brasileira, v.35, p.277-288, 2000.

AZEVEDO, D.M.P. de; NASCIMENTO, H.T.S. do. Potencial forrageiro de espécies para cultivo no período de safrinha em solos de tabuleiros costeiros. Teresina: Embrapa Meio-Norte, 2002. 4p. (Embrapa Meio-Norte. Comunicado Técnico, 148).

BARTZ, H. Dinâmica dos nutrientes e adubação em sistema de produção sob plantio direto. In: FRIES, M.R. Plantio direto em solos arenosos: alternativas de manejo para a sustentabilidade agropecuária. Santa Maria: UFSM; Pallotti, 1998. p.52-63.

BRAZ, A.J.B.P.; SILVEIRA, P.M. da; KLIEMANN, H.J.; ZIMMERMANN, F.J.P. Acumulação de nutrientes em folhas de milheto e dos capins braquiária e mombaça. Pesquisa Agropecuária Tropical, v.34, p.83-87, 2004.

CHAVES, J.C.D.; CALEGARI, A. Adubação verde e rotação de culturas. Informe Agropecuário, v.22, p.53-60, 2001.

DUDA, G.P.; GUERRA, J.G.M.; MONTEIRO, M.T.; DE-POLLI, H.; TEIXEIRA, M.G. Perennial herbaceous legumes as live soil mulches and their effects on $\mathrm{C}, \mathrm{N}$ and $\mathrm{P}$ of the microbial biomass. Scientia Agricola, v.60, p.139-147, 2003.

EMBRAPA. Centro Nacional de Pesquisa de Solos. Manual de métodos de análise de solo. 2.ed. Rio de Janeiro: Embrapa-CNPS, 1997. 212p.

EMBRAPA. Centro Nacional de Pesquisa de Solos. Sistema brasileiro de classificação de solos. Brasília: Embrapa Produção de Informação; Rio de Janeiro: Embrapa Solos, 1999. 412p.

FRANCISCO, E.A.B. Antecipação da adubação da soja na cultura de Eleusine coracana (L.) Gaertn., em sistema de plantio direto.
2002. 55p. Dissertação (Mestrado) - Universidade de São Paulo, Piracicaba.

MALAVOLTA, E.; VITTI, G.C.; OLIVEIRA, S.A. de. Avaliação do estado nutricional de plantas: princípios e aplicações. 2.ed. Piracicaba: Potafos, 1997. 319p.

MARSCHNER, H. Mineral nutrition of higher plants. 2.ed. San Diego: Academic Press, 1995. 889p.

MORAES, R.N.S. Decomposição das palhadas de sorgo e milheto, mineralização de nutrientes e seus efeitos no solo e na cultura do milho em plantio direto. 2001, 90p. Dissertação (Mestrado) - Universidade Federal de Lavras, Lavras.

OLIVEIRA, T.K.; CARVALHO, G.J.; MORAES, R.N.S. Plantas de cobertura e seus efeitos sobre o feijoeiro em plantio direto. Pesquisa Agropecuária Brasileira, v.37, p.1079-1087, 2002.

PADOVAN, M.P.; ALMEIDA, D.L. de; GUERRA, J.G.M.; RIBEIRO, R. de L.D.; OLIVEIRA, F.L. de; SANTOS, L.A.; ALVES, B.J.R.; SOUTO, S.M. Decomposição e liberação de nutrientes de soja cortada em diferentes estádios de desenvolvimento. Pesquisa Agropecuária Brasileira, v.41, p.667-672, 2006.

PAUL, E.A.; CLARK, F.E. Soil microbiology and biochemistry. San Diego: Academic Press, 1989. 275p.

PRIMAVESI, O.; PRIMAVESI, A.C.; ARMELIN, M.J.A. Qualidade mineral e degradabilidade potencial de adubos verdes conduzidos sobre Latossolos, na região tropical de São Carlos, SP, Brasil. Revista de Agricultura, v.77, p.89-102, 2002.

ROSOLEM, C.A.; CALONEGO, J.C.; FOLONI, J.S.S. Lixiviação de potássio da palha de espécies de cobertura de solo de acordo com a quantidade de chuva aplicada. Revista Brasileira de Ciência do Solo, v.27, p.355-362, 2003.

SILVA, E.C. da; MURAOKA, T.; BUZETTI, S.; VELOSO, M.E. da C.; TRIVELIN, P.C.O. Aproveitamento do nitrogênio (15N) da crotalária e do milheto pelo milho sob plantio direto em Latossolo Vermelho de Cerrado. Ciência Rural, v.36, p.739-746, 2006.

SNEDECOR, G.W.; COCHRAN, W.G. Statistical methods. 8.ed. Ames: Iowa State University Press, 1989. 503p.

TEIXEIRA, C.M.; CARVALHO, G.J. de C.; FURTINI NETO, A.E.; ANDRADE, M.J.B. de; MARQUES, E.L.S. Produção de biomassa e teor de macronutrientes do milheto, feijão-de-porco e guandu-anão em cultivo solteiro e consorciado. Ciência e Agrotecnologia, v.29, p.93-99, 2005.

THOMAS, R.J.; ASAKAWA, N.M. Decomposition of leaf litter from tropical forage grasses and legumes. Soil Biology \& Biochemistry, v.25, p.1351-1361, 1993.

TORRES, J.L.R.; PEREIRA, M.G.; ANDRIOLI, I.; POLIDORO, J.C.; FABIAN, A.J. Decomposição e liberação de nitrogênio de resíduos culturais de plantas de cobertura em um solo de cerrado. Revista Brasileira de Ciência do Solo, v.29, p.609-618, 2005.

WIEDER, R.K.; LANG, G.E. A critique of the analytical methods used in examining decomposition data obtained from litter bags. Ecology, v.63, p.1636-1642, 1982.

Recebido em 16 de abril de 2007 e aprovado em 31 de julho de 2007 